Document downloaded from:

http://hdl.handle.net/10251/50796

This paper must be cited as:

Fernández González, HA.; Rubio Arjona, L.; Reig, J.; Rodrigo Peñarrocha, VM.; ValeroNogueira, A. (2013). Path loss modeling for vehicular system performance and communicaitons protocols evaluation. Mobile Networks and Applications. 18(6):755-765. doi:10.1007/s11036-013-0463-x.

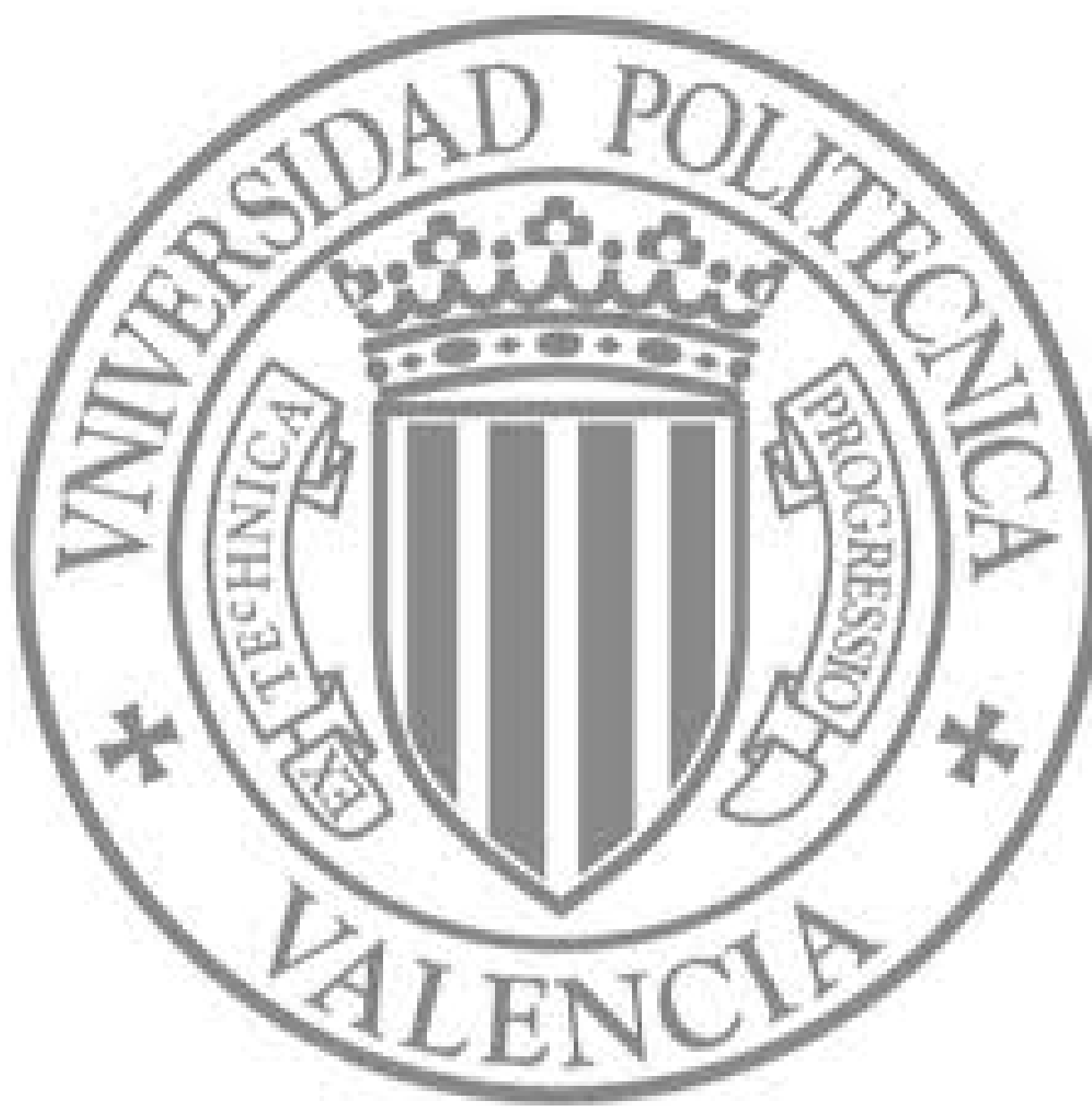

The final publication is available at

http://dx.doi.org/10.1007/s11036-013-0463-x

Copyright Springer Verlag (Germany) 
Ref. \#MONE-S-13-00015

Paper submitted to International Journal of Mobile Networks and Applications.

Special Issue on Networks Protocols and Algorithms for Vehicular Ad Hoc Networks

\title{
Path loss modeling for vehicular system performance and communication protocols evaluation
}

\author{
Herman Fernández • Lorenzo Rubio • Juan Reig • Vicent M. Rodrigo-Peñarrocha • Alejandro \\ Valero
}

\begin{abstract}
Vehicular communications are receiving considerable attention due to the introduction of the intelligent transportation system (ITS) concept, enabling smart and intelligent driving technologies and applications. To design, evaluate and optimize ITS applications and services oriented to improve vehicular safety, but also non-safety applications based on wireless systems, the knowledge of the propagation channel is vital. In particular, the mean path loss is one of the most important parameters used in the link budget, being a measure of the channel quality and limiting the maximum allowed distance between the transmitter (Tx) and the receiver (Rx). From a narrowband vehicular-to-vehicular (V2V) channel measurement campaign carried out at $5.9 \mathrm{GHz}$ in three different urban environments characterized by high traffic density, this paper analyzes the path loss in terms of the Tx-Rx separation distance and fading statistics. Based on a linear slope model, values for the path loss exponent and the standard deviation of shadowing are reported. We have evaluated the packet error rate (PER) and the maximum achievable Tx-Rx separation distance for a PER threshold level of $10 \%$ according to the digital short-range communications (DSRC) specifications. The results reported here can be incorporated in an easy way to vehicular networks (VANETs) simulators in order to develop, evaluate and validate new protocols and systems architecture configurations under realistic propagation conditions.
\end{abstract}

Keywords Vehicular channels, vehicular channel characterization, vehicular communications, vehicular channel measurements, path loss modeling, packet error rate.

\footnotetext{
Herman Fernández

Escuela de Ingeniería Electrónica at the Universidad Pedagógica y Tecnológica de Somagoso,

Colombia

e-mail: herman.fernandez@uptc.edu.co
}

Lorenzo Rubio $(\square)$

Universitat Politècnica de València, Spain

E:mail: lrubio@dcom.upv.es

Juan Reig Pascual

Universitat Politècnica de València, Spain

E:mail: jreigp@dcom.upv.es

Vicent M. Rodrigo-Peñarrocha

Universitat Politècnica de València, Spain

E:mail: vrodrigo@dcom.upv.es

Alejandro Valero

Universitat Politècnica de València, Spain

E:mail: avalero@dcom.upv.es 


\section{Introduction}

During the last years, vehicular communications are receiving considerable attention. The automobile industry, governments and academic institutions have increased economic resources and research efforts oriented to develop new proposals to improve vehicular safety. These new proposals are based on active systems in conjunction to traditional passive systems (e.g., airbags, anti-lock braking system and electronic stability control), promoting the integration of information and communications technologies (wireless, computing and advanced sensor technologies) into both vehicles and the infrastructure along the roadside. These proposals have led to the intelligent transportation system (ITS) concept. At present, different ITS applications have been introduced, such as variable message signs (VMS), located at strategic point (e.g., tunnels and merging highways) to inform drivers about traffic conditions and dangerous situations, navigation systems, cameras and short-range emergency systems [1,2]. Therefore, there are safety applications for distance emergency situations that required large-range vehicular communications systems. In this sense, the introduction of vehicular ad hoc networks (VANETs), as a special case of vehicular communications, can extend the communication distance and provide more information to drivers in real time. Nevertheless, vehicular communications are not only restricted to road safety communications. VANETs also can offer non-safety applications, such as automobile high speed Internet access, in-vehicle entertainment applications, high-speed tolling, real traffic congestion and routing information, among others [3].

Government radio management organizations have allocated specific bands for the deployment of ITS applications. For instance, the Federal Communication Commission (FCC) assigned $75 \mathrm{MHz}$ of licensed spectrum at the $5.9 \mathrm{GHz}$ frequency band (from $5.850 \mathrm{GHz}$ to $5.925 \mathrm{GHz}$ ), as a part of ITS for dedicated short-range communications (DSRC) [4,5]. In Europe, the European Telecommunication Standard Institute (ETSI) adopted the DSRC band for ITS applications assigning $50 \mathrm{MHz}$ (from 5.875 $\mathrm{GHz}$ to $5.925 \mathrm{GHz}$ ) [6]. The European vehicles manufacturers set up the Car-to-Car Consortium (C2CC) [7] to establish and European standard for vehicular communications proposing applications related to the ITS concept.

The special features of both safety and non-safety applications require develop and implement new communication technologies [8-10], where one of the many challenges to be addressed is the characterization and modeling of the vehicular propagation channel [2,3,8]. Thus, the vehicular channel is at present an important area in channel modeling. The motion of both terminals, the transmitter (Tx) and the receiver $(\mathrm{Rx})$, the use of low elevation antennas and the frequency band operation make that vehicular-to-vehicular (V2V) systems differ from the traditional fixed-to-mobile (F2M) or cellular systems. Thus, channel models developed for F2M systems are not suitable in the deployment of future V2V communication systems, and new channel models are essential to evaluate and validate networking protocols under realistic propagation conditions.

In this paper, based on channel measurements at $5.9 \mathrm{GHz}$ we present a vehicular path loss characterization and propose a simplified propagation model suitable for computer simulations. The channel measurements have been performed in two urban environments, with different road traffic densities and propagation characteristics, and in an expressway environment. Unlike other published results and as novelty, an extensively examination of the model parameters is done in statistical sense. From the mean path loss model derived in the measured environments, the packet error rate (PER) and the maximum achievable Tx-Rx separation distance have been evaluated for a PER threshold level of $10 \%$ according to the digital short-range communications (DSRC) specifications. The path loss model and fading statistics derived from our analysis can be incorporated to VANETs simulators to evaluate and analyze the final performance of safety and non-safety applications under realistic propagation conditions.

This paper is organized as follows: Section 2 gives general aspects related to vehicular channel modeling. Section 3 describes the measurement setup and the measured scenarios. Measurement results and path loss modeling are presented in Section 4. In Section 5 a system performance evaluation is done in terms of the PER and maximum achievable Tx-Rx separation distance. Finally, the conclusions are given in Section 6.

\section{Vehicular channel modeling}

The term channel modeling refers to the approaches, models and the measurement campaigns conducted to understand how the propagation channel impairs and distorts the transmitted signal in any wireless communication and environment. Thus, a channel model is a simplified representation of the propagation channel centered in those aspects and metrics that affect the final performance of the system [2,11]. In practice, the most important parameters of the propagation channel to characterize the final performance of a system are the mean path loss and fading statistics due to the multipath and Doppler effects. Mean path loss describes how the mean power at the Rx varies with the Tx-Rx 
separation distance; whereas the fading statistics describe how the instantaneous received power fluctuates over space, time and frequency. Both parameters condition the final performance of the wireless system in terms of the signal-to-noise plus interference ratio (SNIR).

In vehicular communications, the high mobility of both Tx and Rx terminals and interacting objects (reflectors and/or scatterers), makes that vehicular channels exhibit high temporal variability (timeselectivity). Also, the employment of low elevation antennas together with the movement of the interacting objects, the probability of Tx-Rx link obstruction and multipath effect, condition the maximum allowed distance between the Tx and Rx, particularly in dense traffic urban environments. Thus, accurate models of the propagation channel are essential to develop, evaluate and validate new protocols and system architecture configurations. Vehicular networks simulators require the integration of realistic propagation channels, to which may be added also mobility models to take into account the behavior of vehicles. It is worth noting that in the context of vehicular networks, simulations are particularly important due to the difficulty and enormous effort to carry out real tests involving the high number of vehicles, driving conditions and different vehicular environments.

The propagation channel is often investigated by (i) deterministic, (ii) geometric-based stochastic (GBS) or (iii) stochastic approaches [2]. Deterministic channel models characterize the physical propagation channel using a geographical description of the environment and approximation of ray techniques based on the geometrical optic theory (e.g., ray-tracing or ray-launching). Deterministic models allow us to perform realistic simulations of channels. The models can take into account the road traffic and the environment nearby to the road line. Nevertheless, the high computational load, the characteristics of vehicular environments and the resulting large combinations of real propagation conditions, make difficult the development of vehicular channel deterministic models with certain accuracy. GBS models assume a stochastic (or randomly) distribution of interacting objects around the Tx and Rx positions and then performing a deterministic analysis. To reduce computational load, simplified ray-tracing techniques can be incorporated, and to reduce the complexity of the model, it can be assumed that the interacting objects are distributed in regular shapes (e.g., rings or ellipses around the Tx, Rx or both). Stochastic models describe the channel parameters behavior in a stochastic way, without knowledge of the environment geometry, and are based on measurement campaigns. Stochastic models can describe the behavior or the path loss and fading statistics in an easy manner and can be incorporated to network simulators with reduced computational load. It is worth noting that the path loss model derived under a stochastic approach is based on the specific conditions during the measurement campaign. Thus the specific position and number of surrounding interacting objects are taken into account by the model parameters.

For a channel point of view, the main features of the vehicular environment which are necessary to take into account are (i) the type of propagation link, (ii) type of environment (i.e., urban, suburban, expressway, highway, etc.), (iii) vehicles speed, (iv) vehicular traffic density, and (v) direction of motion of the Tx and Rx vehicles (the same or in opposite directions). Although some of the above features are overlapped, e.g., traffic densities are usually higher in urban environments and higher vehicles speed are given in expressway and highway environments, these features increase the possible combinations of real propagation conditions, making difficult the development of deterministic models. For this reason, the characteristics of vehicular propagation channels are often examined by means of simulation models or through measurement campaigns.

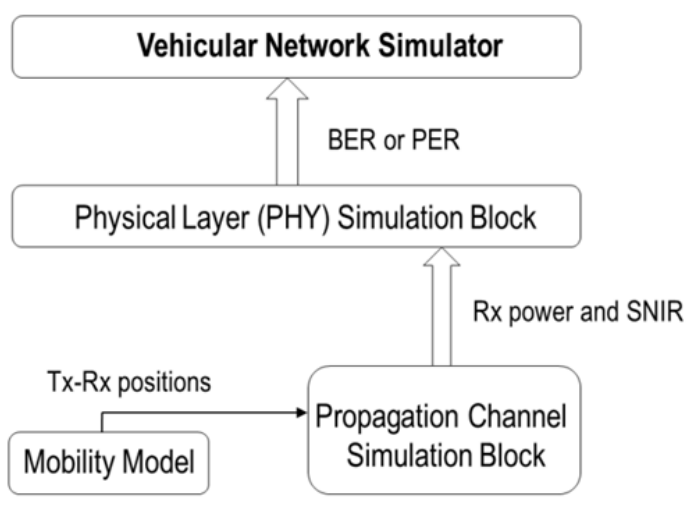

Fig. 1 Simplified architecture of a vehicular network simulator to illustrate the propagation channel simulation block. 
A network simulator uses a propagation channel simulation block (see Fig. 1). For a given Tx-Rx separation distance (Tx-Rx positions), that can be provided by the integration of a vehicular mobility model, the propagation channel simulation block can estimate the Rx power and the SNIR based on the mean path loss prediction. The propagation model implemented can take into account the antennas height and their radiation patters, the type of vehicles and the characteristics of the propagation environment. A physical layer (PHY) simulation block can estimate the bit error rate (BER) or the corresponding packet error rate (PER). In vehicular networks the PHY simulator block is based on the IEEE 802.11p standard [12], which is an adaptation of the IEEE 802.11a standard. The network simulator, based on the performance metrics derived by the PHY simulator estimates and evaluates the performance of the system taking into account the metrics required in safety and non-safety applications and services, such as delivery ratio, throughput and latency (delay).

\section{Vehicular channel measurements}

\subsection{Measurement setup and procedure}

The measurement setup used to perform the narrowband channel measurements is shown in Fig. 2. A HP83623A Signal Generator (SG) was used at the Tx side. The SG transmitted a continuous wave (CW) at $5.9 \mathrm{GHz}$ (the DSRC band) with $-10 \mathrm{dBm}$. A high power amplifier (HPA) allowed us to transmit with $+23.8 \mathrm{dBm}$ of EIRP (Equivalent Isotropically Radiated Power). Fig. 3(a) shows the measurement setup on-board at the Tx vehicle. On the other hand, at the Rx side a ZVA24 Rohde \& Schwarz Vector Network Analyzer (VNA), configured as a power meter ( $0 \mathrm{~Hz}$ of SPAN and central frequency equal to $5.9 \mathrm{GHz}$ ), was used to measure the received signal level. A laptop controlled the VNA to automate the measurements acquisition system and record the measured data. Furthermore, we used two medium high power amplifiers (MPAs) at the Rx and low-loss cables $(1.15 \mathrm{dBm} / \mathrm{m}$ at 5.9 $\mathrm{GHz}$ ), to achieve a total gain system of about $86.67 \mathrm{~dB}$. A view of the devices on-board at the $\mathrm{Rx}$ vehicle is shown in Fig. 3(b).
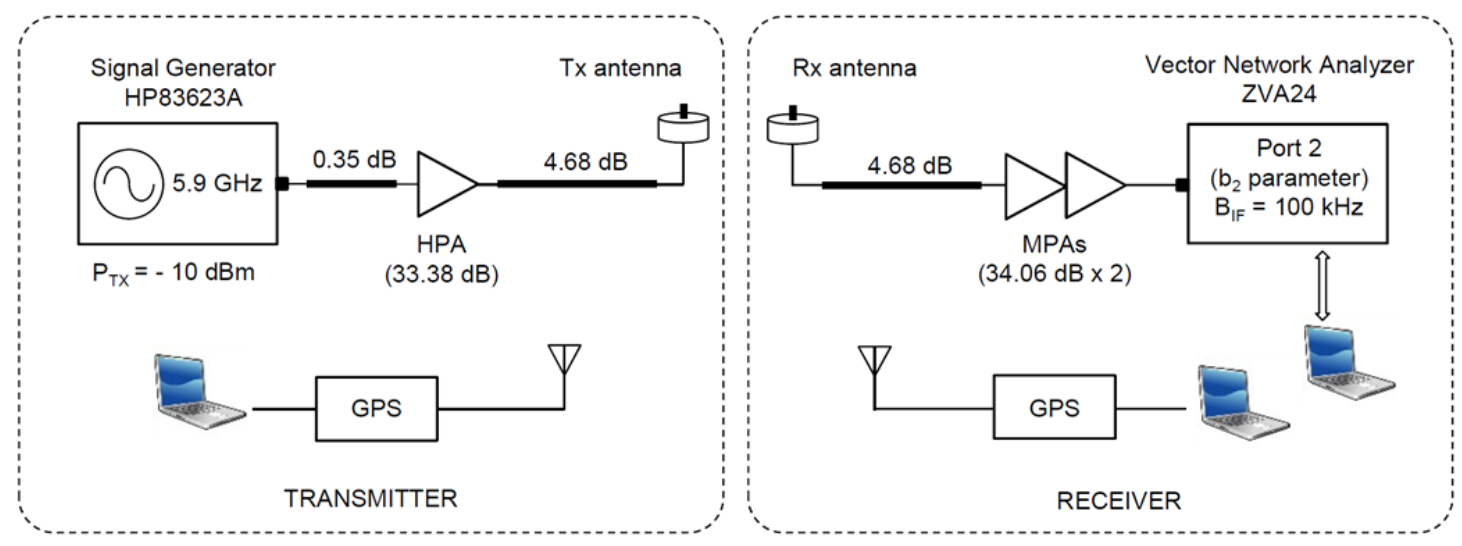

Fig. 2 Measurement setup diagram.

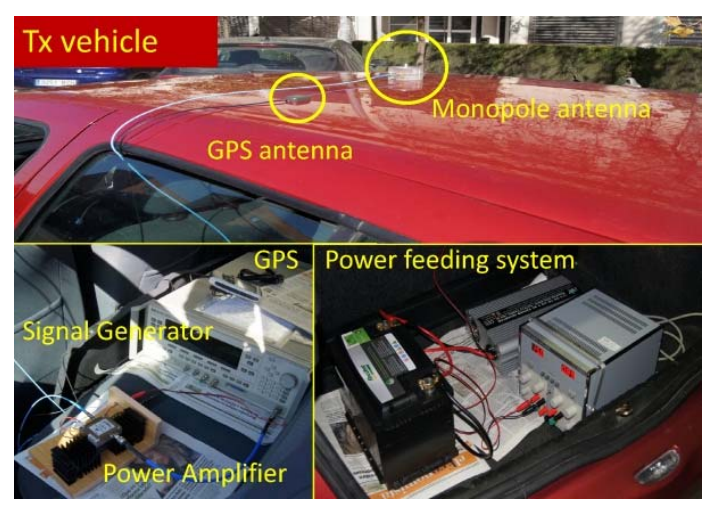

(a)

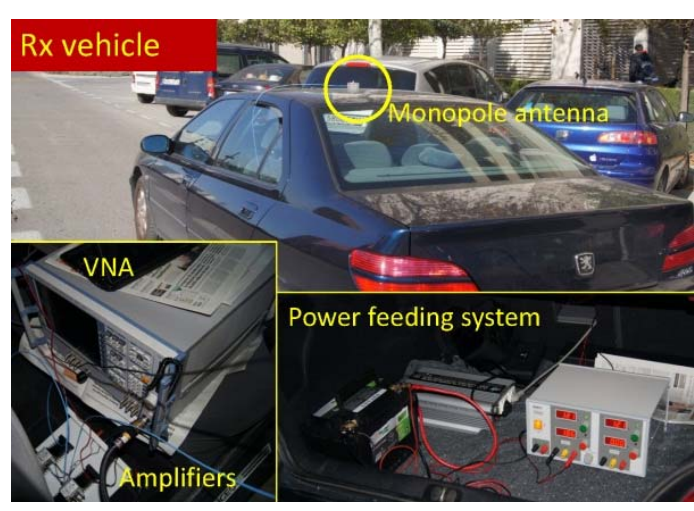

(b)

Fig. 3 View of the devices on-board at the vehicles: (a) Tx vehicle and (b) Rx vehicle. 
During the measurement campaign, the VNA measured the received signal level measuring directly the $\mathrm{b}_{2}$ parameter, i.e., the output of the last amplifier at the Rx was connected to the port 2 of the VNA. It is worth noting that the VNA has independent receivers connected to the ports, with high selectivity and sensitivity. The $\mathrm{b}_{2}$ parameter was continuously measured using traces of $N$ test points. Fig. 4 shows the acquisition process of the measurements. Each trace requires a sweep time and then a record time. The averaging of the measured samples in each trace permits us to remove (filtering) the fast fading fluctuations of the received signal level. This last is equivalent to consider an integration period in each power measurement equal to the time sweep per trace. Thus, the fluctuations of the measured signal are due to shadowing and blocking effects of the interacting vehicles between and around the Tx and Rx.

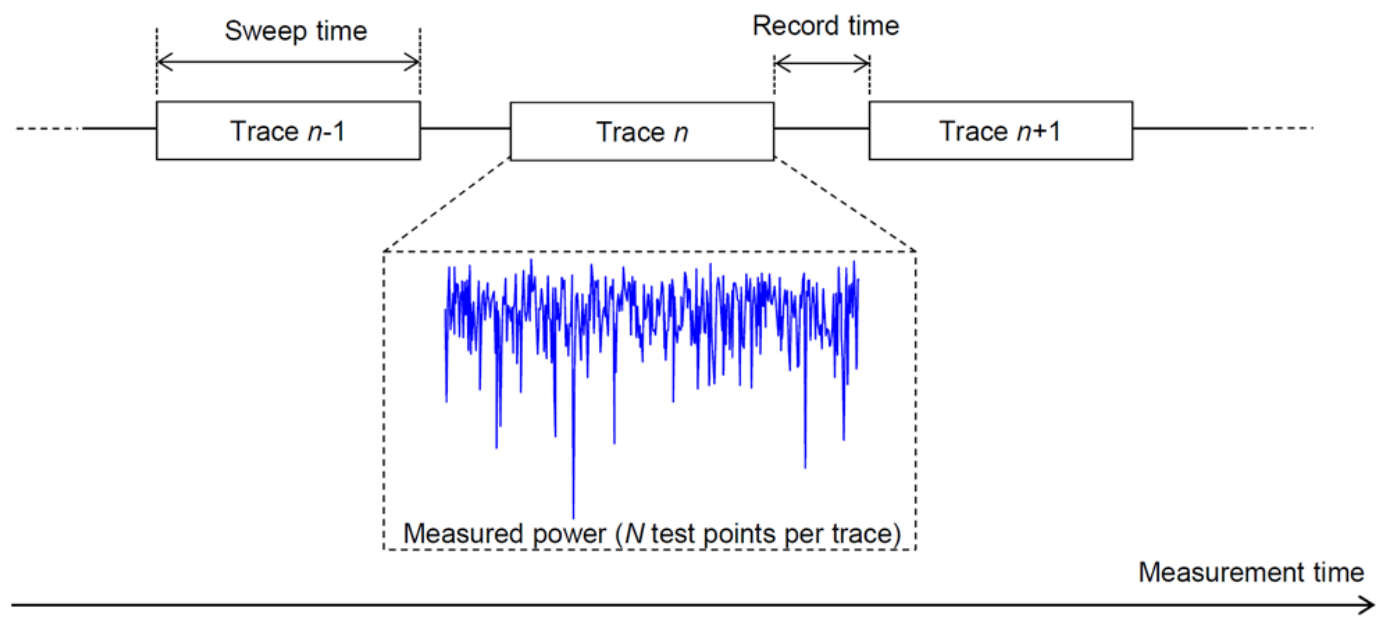

Fig. 4 Acquisition process of the measurements at the VNA.

We have taken into account that both the thermal noise level at the Rx and the measurement acquisition time of the VNA are related to the value of the Bandwidth of the VNA's Intermediate Filter, denoted by $B_{I F}$. Thus, a low value of $B_{I F}$ decreases the noise level and therefore permits us to perform measurements with a greater Tx-Rx separation distance. Whereas a high value of $B_{I F}$ reduces the acquisition time improving the spatial resolution of the measurements. As a compromise, we decided to choose $100 \mathrm{kHz}$ as $B_{I F}$, yielding a noise level around $-70 \mathrm{dBm}$ and an average acquisition time per test point of $45 \mu \mathrm{s}$. This value of $B_{I F}$ is greater than the maximum Doppler frequency in vehicular environments [2], where there is a maximum Doppler frequency of about $1 \mathrm{kHz}$ for a relative speed of $55 \mathrm{~m} / \mathrm{s}(198 \mathrm{~km} / \mathrm{h})$. In the measurement campaign the number of test points per trace was 5000 , resulting in an average sweep time per trace of about $220 \mathrm{~ms}$ and a record time of $25 \mathrm{~ms}$. With this configuration, the integration period of the measured signal level is equivalent to a vehicle displacement of $60 \lambda$ at $13.88 \mathrm{~m} / \mathrm{s}(50 \mathrm{~km} / \mathrm{h})$.

Both Tx and Rx used the same antenna, a $\lambda / 4$ monopole with a maximum gain of about $5.44 \mathrm{~dB}$ and a $\mathrm{S}_{11}$-scattering parameter lower than $-21 \mathrm{~dB}$ at $5.9 \mathrm{GHz}$. The gain and the radiation pattern were measured in an anechoic chamber, mounting the antenna over a $1 \mathrm{~m}$ by $1 \mathrm{~m}$ metallic plane emulating the root of a car. A view of the antenna and its radiation pattern in the elevation plane is shown in Fig. 5. In order to achieve an omnidirectional coverage, the antennas were roof-mounted in the center of the vehicles (see Fig. 2) through a magnetic base and transmitting with vertical polarization. The roofposition of the antennas ensures reasonable propagation properties for all possible multipath contributions in the horizontal plane (i.e., possible combinations of angles of departure and incidence at the Tx and Rx, respectively). The measured gain of the antennas in the horizontal plane was $-2.56 \mathrm{~dB}$. 


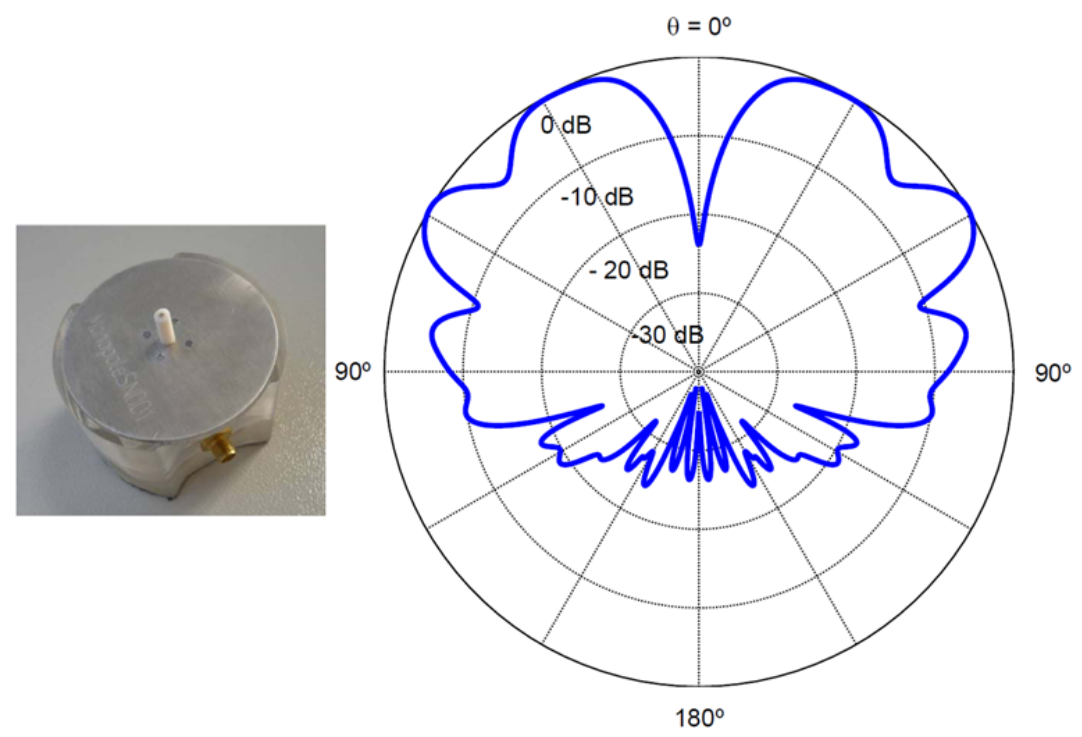

Fig. 5 View of the monopole antenna used in the measurements and its radiation pattern in the elevation plane. The radiation pattern has been measured with the antenna mounted over a finite metallic plane with $1 \mathrm{~m}$ by $1 \mathrm{~m}$ dimensions.

In addition to the RF devices, the Tx and Rx vehicles were equipped with a GPS (Global Positioning System) receiver, each one controlled by a laptop, to provide constant information about the acquisition time of the measurements, as well as relative speed and separation distance between the Tx and Rx vehicles. The laptops were synchronized over time to relate the data from the different GPSs and the measurements from the VNA.

Since the measurement system was installed in vehicles, a power feeding system was needed. The power was achieved from a 75 Ah battery. It supplied $12 \mathrm{~V}$-DC and then, by using an inverter, a 220 $\mathrm{V}$-AC was obtained, to provide around 90 minutes of autonomy.

\subsection{Measurement scenarios}

We have performed measurements at three different scenarios: (i) urban with high traffic density, denoted as scenario 1, (2) urban whit low traffic density, denoted as scenario 2, and (3) expressway with high traffic density, denoted as scenario 3. A brief description of the measured scenarios is the following:

- Scenario 1: This environment corresponds to a large avenue of the urban area of the city of Valencia, Spain, with five lanes and characterized by high road traffic density (an average traffic density around 29590 vehicles/24h as published by the City Council). It is one-way travel avenue, which ends at the port. The avenue width is about $26.5 \mathrm{~m}$, including a lane of parked cars on both sides and the sidewalks. The total length of the measured route was about 2773 m. Fig. 6(a) shows a view from the Tx vehicle of this scenario.

- Scenario 2: This environment corresponds to an urban area of Valencia characterized by low road traffic density (an average traffic density around 7440 vehicles/24h as published by the City Council) and one or two lanes of one-way travel direction. The average street width of the urban area is about $10 \mathrm{~m}$, including a lane of parked cars on both sides and the sidewalks. The total length of the measured route was about $1410 \mathrm{~m}$. Fig. 6(b) shows a view from the Tx vehicle of this scenario.

- Scenario 3: This environment corresponds to an expressway north of Valencia, with high road traffic density (we have not data of the road traffic density) and three lanes in each direction. Both sides of the expressway are open areas. The total length of the route measured was 14379 m. Fig. 6(c) shows a view from the Tx vehicle of this scenario. 


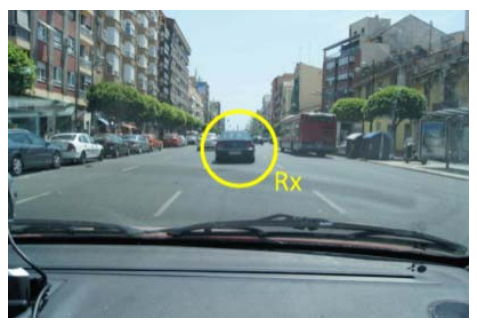

(a)

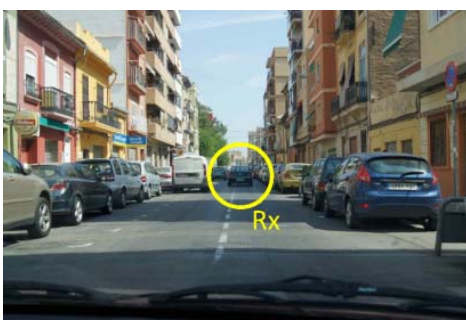

(b)

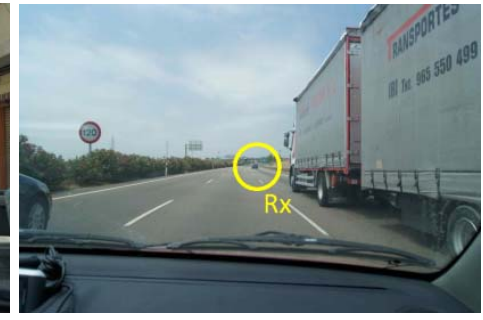

(c)

Fig. 6 Views of the measurement scenarios from the Tx vehicle: (a) scenario 1, (b) scenario 2, and (c) scenario 3.

For scenarios 1 and 2 the speed limit is $13.88 \mathrm{~m} / \mathrm{s}$ (50 km/h), whereas for scenario 3 is $33.33 \mathrm{~m} / \mathrm{s}$ (120 $\mathrm{km} / \mathrm{h}$ ). For each scenario, the measurements were taken between 11:00 a.m. and 12:30 p.m., with the Tx and Rx vehicles in the same direction and in real driving and traffic conditions, predominating the blockage of the line-of-sight (LOS) due to the vehicles between the Tx and Rx link, especially when the Tx-Rx separation distance increases in scenario 3. In our study we have not distinguished between LOS and non-LOS (NLOS) conditions. During the measurements, the Tx and Rx vehicles traveled at different separations and speeds according to the road traffic conditions. Also, we have tried to vary the Tx-Rx separation distance to have enough measured data to analyze the path loss in terms of the separation distance between terminals.

The vehicles involved in the measurements campaign were a Renault Clio as Tx and a Peugeot 406 as $\mathrm{Rx}$. The antennas heights, mounted in the roof-center of the vehicles, were $1.41 \mathrm{~m}$ and $1.43 \mathrm{~m}$ above the ground for the Tx and the $\mathrm{Rx}$, respectively.

\section{Measurement results}

Fig. 7 shows the scatter plot of the path loss as a function of the Tx-Rx separation distance in a log scale from measured data. The red line corresponds to the best fit to the measured data using the leastsquares regression procedure. It can be observed that there is a linear relationship between the path loss and the logarithmic of the Tx-Rx separation distance. Thus, the path loss can be described in identical manner as the traditional F2M channels by the classical formula [2,8]:

$$
P L(d)=P L_{0}+10 \gamma \log _{10}\left(d / d_{0}\right)+X, \quad d \geq d_{0},
$$

where $d$ is the distance between the Tx and Rx, $P L_{0}$ is the path loss at the reference distance $d_{0}, \gamma$ is the path loss exponent related to the propagation environment, and $X$ is a zero mean random variable with normal distribution and standard deviation $\sigma_{X}$, used to model the large scale fading (due shadowing and blocking effects). For some environments, e.g., indoor environments, it is usual to establish $d_{0}=1 \mathrm{~m}$, thus the term $P L_{0}$ refers to the path loss for a Tx-Rx separation distance equal to 1 m. Nevertheless, in vehicular environments and for empirical path loss characterization it is more convenient to write Eq. (1) in the form

$$
P L(d)=P L_{0}^{*}+10 \gamma \log _{10}(d)+X, \quad d \geq d_{\min },
$$

where the term

$$
P L_{0}^{*}=P L_{0}-10 \gamma \log _{10}\left(d_{0}\right)
$$

takes into account a reference distance related to the measurements and then the linear model is restricted to distances exceeding $d_{\min }$, which is the minimum Tx-Rx separation distance for the measured data. In our study $d_{\text {min }}$ is lower than $10 \mathrm{~m}$ (e.g., see Fig. 7 where $d_{\min }$ ranges from 5 to $8 \mathrm{~m}$ ). Eq. (3) indicates that the constant term in $P L(d)$ given by (2), $P L_{0}^{*}$, is related to both the path loss at the reference distance $d_{0}, P L_{0}$, and the path loss exponent. This relationship will be discussed later. 

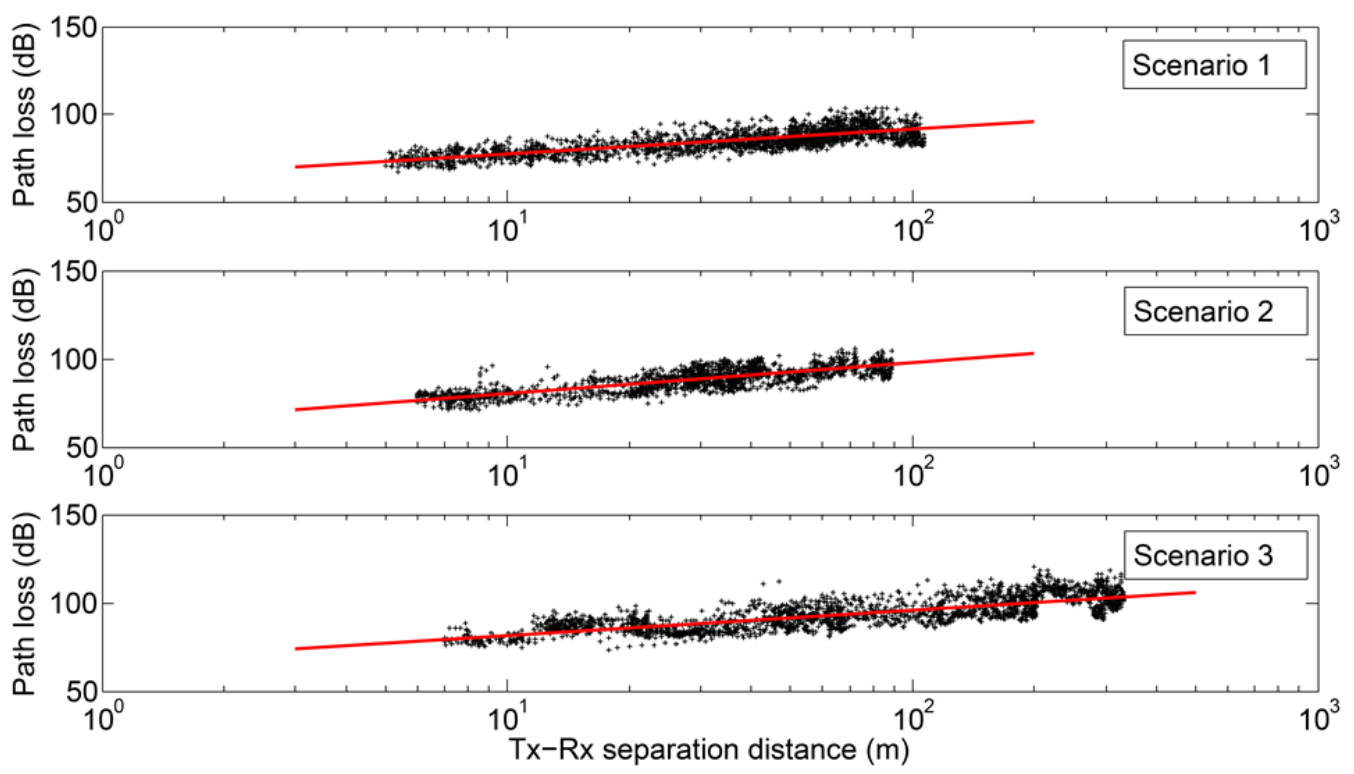

Fig. 7 Scatter plot of the path loss as a function of the Tx-Rx separation distance. The + mark indicates measured data and the red line indicates linear fit.

To perform a statistical characterization of the linear path loss model proposed by (2), we have processed the measured data using a sliding window method. We have set a window size containing 500 samples of the mean received power. A sample of mean received power corresponds to the averaging of all test points measured per trace (See Fig. 4). This window size is equivalent to a time period of about $120 \mathrm{~s}$ (500 consecutive traces). The parameters of the path loss model have been estimated in a least-squares sense for each window, sliding the window from the beginning to the end of the measured data records for each scenario. Thus, for a record of $M$ traces measured, the number of windows where the parameters of the model are estimated is $M-500$. Table 1 summarizes the minimum, mean, maximum and standard deviation (Std. dev) of the linear path loss model parameters. For example, in scenario $1 P L_{0}^{*}$ ranges from $57.51 \mathrm{~dB}$ to $67.15 \mathrm{~dB}$, with a mean value of $61.98 \mathrm{~dB}$ and a standard deviation of $1.99 \mathrm{~dB}$; the path loss exponent ranges from 1.19 to 1.73 , with a mean value of 1.47 and a standard deviation of 0.12 . The term $\sigma_{X}$ which describes the large scale fluctuations ranges from $2.12 \mathrm{~dB}$ to $2.95 \mathrm{~dB}$, with a mean value of $2.47 \mathrm{~dB}$ and a standard deviation of $0.23 \mathrm{~dB}$. Mean values of $P L_{0}^{*}$ and the path loss exponent are $61.55 \mathrm{~dB}$ and 1.84, and $58.37 \mathrm{~dB}$ and 1.98 for scenarios 2 and 3 , respectively.

\begin{tabular}{|l|l|r|r|c|}
\cline { 2 - 5 } \multicolumn{1}{c|}{} & Parameter / Value & $P L_{0}^{*}(\mathrm{~dB})$ & $\gamma$ & $\sigma_{X}(\mathrm{~dB})$ \\
\hline \hline \multirow{3}{*}{ Scenario 1 } & Maximum & 67.15 & 1.73 & 2.95 \\
\cline { 2 - 5 } & Mean & 61.98 & 1.47 & 2.47 \\
\cline { 2 - 5 } & Minimum & 57.51 & 1.19 & 2.12 \\
\cline { 2 - 5 } & Std. dev & 1.99 & 0.12 & 0.23 \\
\hline \hline \multirow{3}{*}{ Scenario 2 } & Maximum & 67.20 & 2.66 & 2.53 \\
\cline { 2 - 5 } & Mean & 61.55 & 1.84 & 2.33 \\
\cline { 2 - 5 } & Minimum & 51.05 & 1.45 & 1.98 \\
\cline { 2 - 5 } & Std. dev & 2.41 & 0.22 & 0.13 \\
\hline \hline Scenario 3 & Maximum & 69.66 & 3.07 & 4.49 \\
\cline { 2 - 5 } & Mean & 58.37 & 1.98 & 3.97 \\
\cline { 2 - 5 } & Minimum & 38.50 & 1.33 & 3.55 \\
\cline { 2 - 5 } & Std. dev & 7.68 & 0.40 & 0.26 \\
\hline \hline
\end{tabular}

Table 1 Linear path loss model parameters for the three measured scenarios. 
From the results of Table 1, the greatest large scale fading fluctuations occur in expressway, where the vehicles speed is higher and the blocking effect of the Tx-Rx link is more probable. Also, the path loss exponent takes larger values, with a mean value of 1.98 , whereas the $P L_{0}^{*}$ term is lower, with a mean value of $58.37 \mathrm{~dB}$. Comparing scenarios 1 and 2, higher values of the path loss exponent are derived in the scenario 2, whereas the large scale fading fluctuations are lower. It is worth noting that the road traffic density in scenario 2 is lower and then there are less interacting objects.
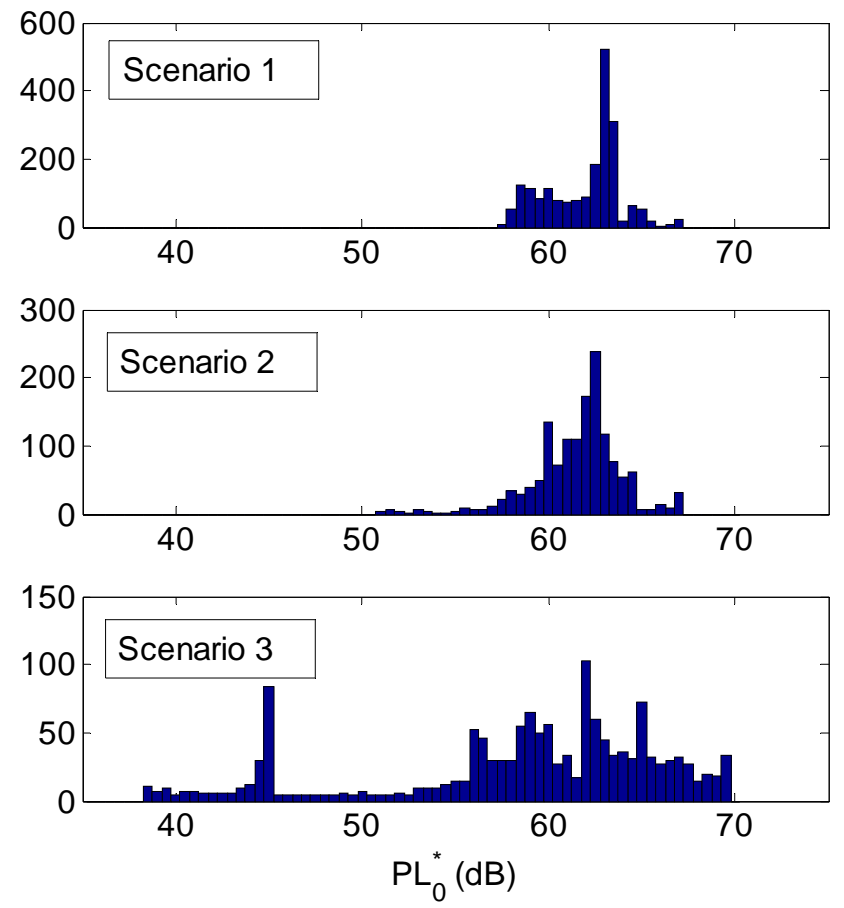

Fig. 8 Histogram of the $P L_{0}^{*}$ term for the three measured scenarios.
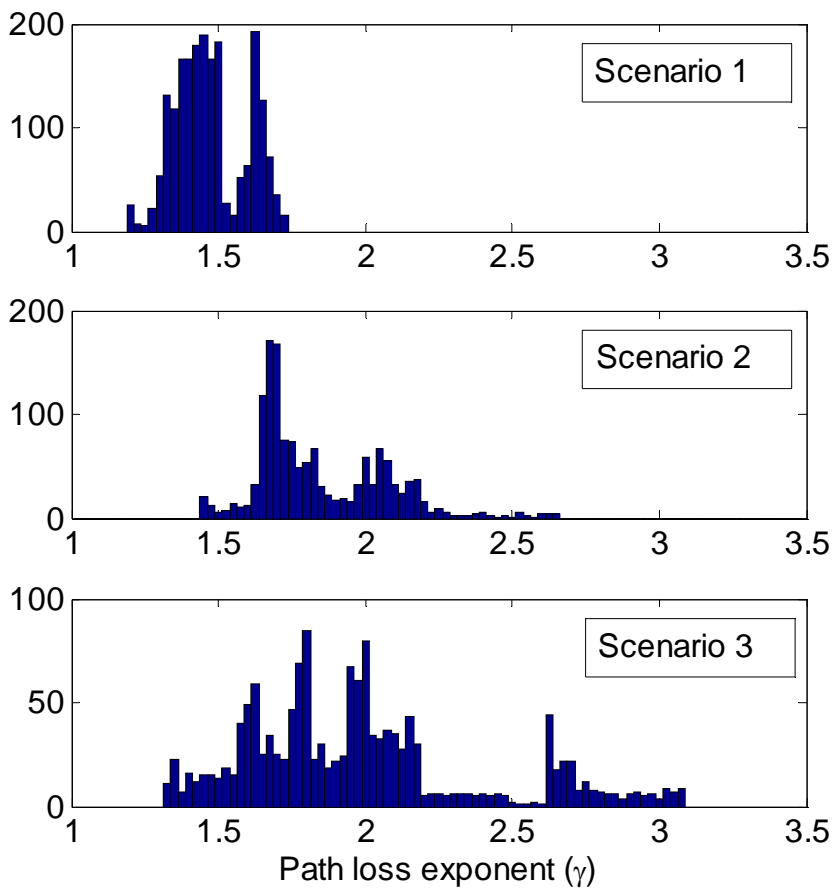

Fig. 9 Histogram of the path loss exponent for the three measured scenarios. 
To analyze the statistical dispersion of the propagation model parameters, Figs. 8 and 9 show the histogram of the $P L_{0}^{*}$ term and the path loss exponent, respectively. In order to facilitate comparisons, the abscissa axis covers the same range in each figure. From Fig. 8, the higher dispersion of $P L_{0}^{*}$ occurs in scenario 3. In the three scenarios, the most frequent value (i.e., the mode of the statistical distribution) is almost the same, about $63 \mathrm{~dB}$, which is greater than the path loss for LOS propagation conditions ( $47.85 \mathrm{~dB}$ at $5.9 \mathrm{GHz}$ for $1 \mathrm{~m} \mathrm{Tx}-\mathrm{Rx}$ separation distance). Nevertheless, in scenario 3 is also frequent a value of about $45 \mathrm{~dB}$, showing that there were LOS conditions during the measurements with constructive multipath interference. According to the results shown in Fig. 8, the greater dispersion again occurs in scenario 3 , where there are path loss exponent values greater than 2.5. Due to the changes in the propagation conditions related to the driving behavior and the movement of the scatterers, in the expressway environment there are some frequent values, i.e., 1.62, 1.79 and 1.98, whereas the distribution of the path loss parameter in scenarios 1 and 2 exhibits a bimodal behavior with a common value of about 1.65 .

Contrary to what one might think, the path loss exponent values in scenario 1 are lower than those derived in scenario 2, where the propagation conditions are more favorable, and lower than the values for LOS conditions. To explain this behavior it is necessary to take into account the relation between $P L_{0}^{*}$ and the path loss exponent given by (3). The greater $P L_{0}^{*}$, the lower path loss exponent and vice versa. We have observed from the measurement data that a path loss exponent lower than 2 not necessarily corresponds to propagation conditions better than free space. Generally, values of the path loss exponent lower than 2 are related to $P L_{0}^{*}$ greater than $47.85 \mathrm{~dB}$ ( $P L_{0}$ in free space). This means that although the path loss exponent is lower than 2, the total path loss is greater than the path loss in LOS conditions, as expected. Fig. 10 shows the scatter plot of the path loss exponent as a function of $P L_{0}^{*}$. The linear correlation coefficient between both parameters is $0.94,0.91$ and 0.98 for scenarios 1 ,

2 and 3, respectively. This relationship between $P L_{0}^{*}$ and the path loss exponent agrees with the values reported in [13], where values of the path loss exponent lower than 2 were also measured.

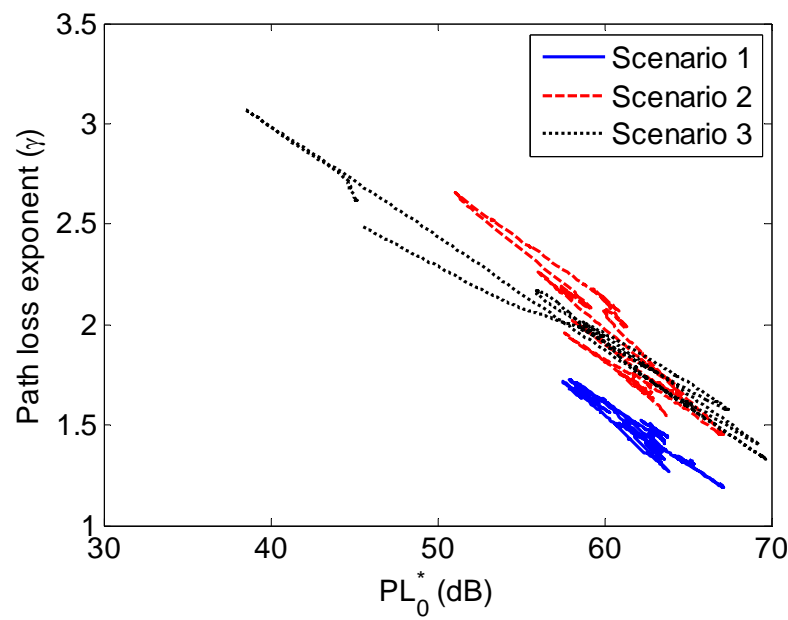

Fig. 10 Scatter plot of the relationship between the path loss exponent and the $P L_{0}^{*}$ parameter for the three measured scenarios.

The values of the linear path loss model summarized in Table 1 and in the histograms of Figs. 8 and 9 permit us to analyze and evaluate the impact of the mean path loss on the vehicular system performance in urban and expressway environments. Unlike other publish results [13-18], we have derived a range of values for the linear path loss model, not only mean values. The mean value of the path loss exponent derived in scenario 1 (1.47) is slightly lower than the values reported in [13] and [18] for urban environments, where values of 1.61 and 1.68 were measured, respectively. By the contrary, the value in scenario $2(1.84)$ is greater. The value of the path loss exponent in scenario 3 (1.98) is close to the values reported in [14] and [18] for highway environments, where values of 1.85 and 1.9 were measured, respectively. With respect to the $\sigma_{X}$ values, our results are in the same order to the published, except for the results reported in [14] where the values are higher. In all cases, the results published in the literature are within the range of our measured data for both the path loss exponent and $\sigma_{X}$. 
It is worth noting that the values of the model parameters are based on the specific conditions during the measurement campaign. In this sense the propagation conditions, e.g., the position and number of surrounding scatterers, are model by the $X$ variable and its standard deviation $\sigma_{X}$. Since large differences exist among cities, expressways and highways around the world, and even in a country, more measurement campaigns must be conducted in different places to have a better knowledge of the vehicular propagation channel.

\section{System performance evaluation}

In vehicular networks, the final performance of road safety applications is mainly influenced by the PER that is conditioned by both the mean path loss and the SNIR. In this section we will evaluate the PER and derive the maximum Tx-Rx separation distance, i.e., the link communications range, under the propagation conditions exhibited by the measured scenarios. We will use the mean value of the propagation model parameters to derive the mean path loss. We have focused our attention on the control channel of the protocol stack standardized for DSRC systems, also known as wireless access in vehicular environments (WAVE), whose PHY is the IEEE 802.11p standard [12]. The control channel is used as the reference channel to initiate and establish all communications. This channel transmits periodically in broadcast mode the available application services, warning messages and safety status messages, among others. The effects of the media access control (MAC) data and PHY protocols of the DSRC/IEEE 802.11p specifications have been taken into account by means of the look-up table (LUT) reported in [19]. This LUT was extracted by link-level simulations in [20], mapping the PER to the channel quality conditions expressed in terms of an "effective" SNIR, denoted by $E_{a v} / N_{0}$. According to [20], $E_{a v} / N_{0}=\alpha S N I R$, where the $\alpha$ parameter takes into account the effect introduced by the cyclic prefix attached to each OFDM (Orthogonal Frequency-Division Modulation) symbol and takes a value equal to 0.8 .

In our analysis we have considered an EIRP for the Tx on-board unit (OBU) of $33 \mathrm{dBm}$, which corresponds to the maximum value according to the DSRC specifications for private applications, a receive antenna gain equal to $-3 \mathrm{~dB}$, and a background noise of $-90 \mathrm{dBm}$ (the background takes into account both the thermal noise and the interference [19]). The LUT corresponds to a QPSK (Quadrature Phase Shift Keying) modulation, a coding rate of 1/2, and packet sizes (PSs) of 39 B, 275 $\mathrm{B}$ and $2304 \mathrm{~B}$. Based on the mean values of the linear path loss model parameters derived from the measured scenarios, the SNIR at the receiver is estimated and then the final PER is obtained by means of the LUT. Fig. 11 shows the PER in terms of the Tx-Rx separation distance for different PSs. It can be seen that for a fixed Tx-Rx separation distance, the PER increases with the PS. Comparing the curves of PER, scenarios 2 and 3 produce a similar performance, whereas scenario 1 shows the best performance because it has smaller path loss exponent values compared to scenario 3 and a smaller $P L_{0}^{*}$ term than scenario 1 , i.e., the mean path loss in scenario 1 is lower for a fixed Tx-Rx separation distance.

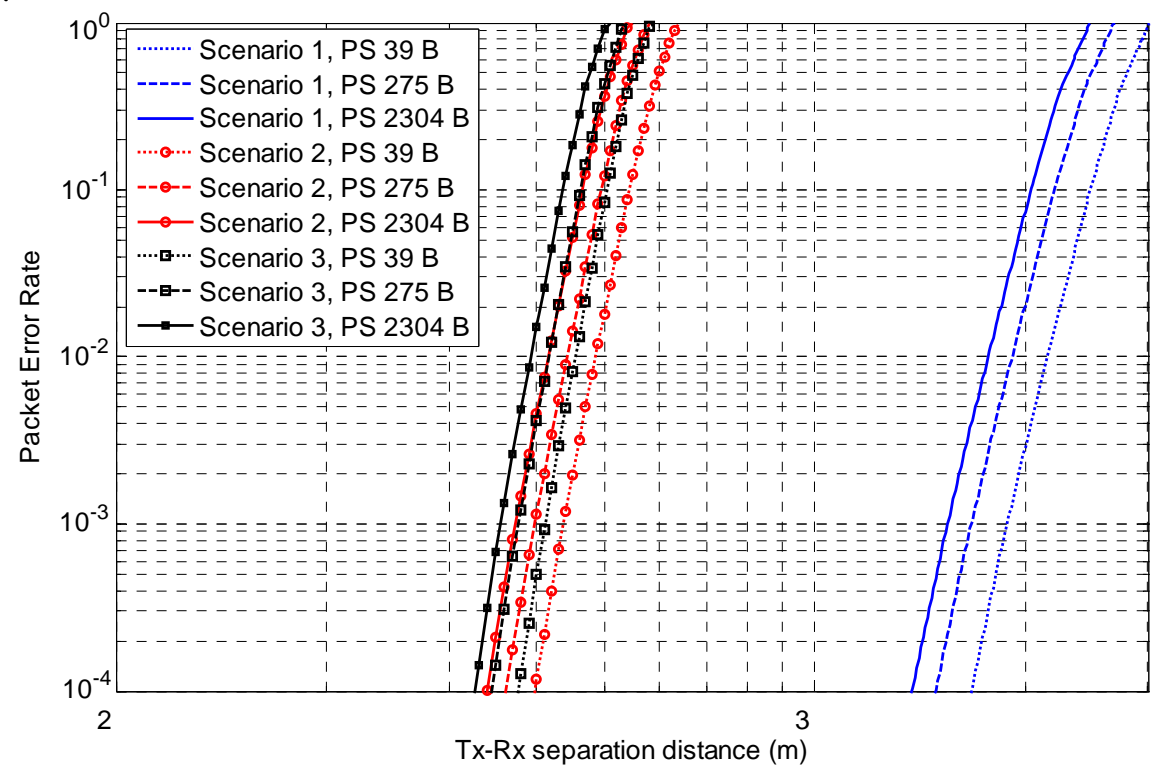

Fig. 11 Packet Error Rate (PER) probability for the WAVE control channel for the measured scenarios. A packet size (PS) equal to 39 B, 275 B and 2304 B has been considered. 
For a PER threshold equal to $10 \%$, Table 2 summarizes the maximum achievable Tx-Rx separation distance. Mean Tx-Rx separation distances slightly greater than $500 \mathrm{~m}$ can be achieved in scenarios 2 and 3 for a PS of $39 \mathrm{~B}$. The mean Tx-Rx separation distance increases until $2478 \mathrm{~m}$ in scenario 1 for the same PS. Comparing the minimum and maximum values of the achievable Tx-Rx separation distances, there are large differences highlighting the heavily impact of the propagation conditions on the final system performance. Thus, the difference between the maximum and minimum Tx-Rx links are greater than $1000 \mathrm{~m}$ for scenarios 2 and 3, reaching a value greater than $4500 \mathrm{~m}$ in scenario 1 for a PS of $39 \mathrm{~B}$.

It is worth noting that the maximum achievable $\mathrm{Tx}-\mathrm{Rx}$ separation distance for expressway is reasonable, whereas in urban environments distances greater than $500 \mathrm{~m}$ are not possible in practice due to the planimetry or geographical distribution of streets. Only in large avenues can be expected greater Tx-Rx separation distance. When there are corners involved in the horizontal propagation, more sophisticated models taking into account diffraction effects are necessary, where corners attenuation higher than 20 or $30 \mathrm{~dB}$ can be achieved.

\begin{tabular}{|l|l|r|c|c|}
\cline { 3 - 5 } \multicolumn{2}{c|}{} & PS 39 B & PS 275 B & PS 2304 B \\
\hline \hline \multirow{3}{*}{ Scenario 1 } & Maximum & 5789 & 4947 & 4519 \\
\cline { 2 - 5 } & Mean & 2478 & 2206 & 2041 \\
\cline { 2 - 5 } & Minimum & 1385 & 1257 & 1171 \\
\hline \hline \multirow{3}{*}{ Scenario 2 } & Maximum & 1206 & 1067 & 988 \\
\cline { 2 - 5 } & Mean & 544 & 495 & 465 \\
\cline { 2 - 5 } & Minimum & 194 & 181 & 173 \\
\hline \hline \multirow{3}{*}{ Scenario 3 } & Maximum & 1493 & 1312 & 1201 \\
\cline { 2 - 5 } & Mean & 503 & 462 & 436 \\
\cline { 2 - 5 } & Minimum & 245 & 232 & 224 \\
\hline \hline
\end{tabular}

Table 2 Tx-Rx separation distance in meters for an average Packet Error Rate (PER) equal to 10\% for the WAVE control channel and for different packet size (PS)

\section{Conclusions}

To address the requirements of safety and non-safety applications and services in VANETs, it is necessary to understand and characterize the propagation characteristics of vehicular channels, especially in those environments with high road traffic densities and mobility of the terminals. Based on a narrowband channel measurement campaign carried out at $5.9 \mathrm{GHz}$ (the DSRC frequency band), we have analyzed the path loss in two urban scenarios and in an expressway environment. We have proposed a path loss model stabling a linear relationship between the path loss and the logarithmic of the Tx-Rx separation distance, in a similar manner to the conventional F2M environments.

The parameters of the path loss model have been examined statistically, reporting a range of values for the parameters of the model. Mean path loss exponent values of 1.47 and 1.84 have been derived from the measured data in the urban environment. For the expressway scenario we have found a mean path loss exponent equal to 1.98. The measured expressway scenario exhibits high dispersion parameters compared to the measured urban environments due to the high mobility of interacting objects and the blocking effects of the Tx-Rx link. Based on the results derived from the measurements, the system performance was examined in terms of the PER and the maximum achievable Tx-Rx separation distance taking into account the DSRC specifications. For the WAVE control channel, the allowed average Tx-Rx separation distance is in the order of $500 \mathrm{~m}$ for a PS equal to $39 \mathrm{~B}$ in the urban (544 m), with low traffic density and narrow streets, and the expressway (503 m) scenarios. For a large and wide avenue, this distance can increase up to $2475 \mathrm{~m}$.

The values reported here for the path loss model parameters can be easily incorporated into VANETs simulators in order to take into account more realistic propagation conditions, and can also be used in the planning and deployment of future vehicular networks. 
Acknowledgements The authors want to thank J. A. Campuzano, D. Balaguer and L. Moragón for their support during the measurement campaign, as well as B. Bernardo-Clemente and A. Vila-Jiménez for their support and assistance in the laboratory activities.

\section{References}

1. Gallager, B., Akatsuka, H., \& Suzuki, H. (2006). Wireless communications for vehicle safety: Radio link performance and wireless connectivity. IEEE Vehicular Technology Magazine, 1(4), 4-24.

2. Rubio, L., Reig, J., \& Fernández, H. (2011). Propagation aspects in vehicular networks, Vehicular technologies. Miguel Almeida (Ed), InTech.

3. Wang, C.-X., Vasilakos, A., Murch, R., Shen, S. G. X., Chen, W., \& - Kosch, T. (2011). Guest Editorial. Vehicular Communications and Networks - Part I, IEEE Journal on Selected Areas in Communications, 29(1), 1-6.

4. ASTM E2213-03 (2003). Standard Specification for Telecommunications and Information Exchange Between Roadside and Vehicle Systems - $5 \mathrm{GHz}$ Band Dedicated Short Range Communications (DSRC) Medium Access Control (MAC) and Physical Layer (PHY) Specifications, American Society for Testing Materials (ASTM), West Conshohocken, PA, USA.

5. IEEE 1609 - Family of Standards for Wireless Access in Vehicular Environments (WAVE). [Online]. Available: http://www.standards.its.dot.gov

6. ETSI TR 102 492-2 Part 2 (2006). Technical Characteristics for Pan European Harminized Communications Equipment Operating in the $5 \mathrm{GHz}$ Frequency Range Intended for Road Safety and Traffic Management, and for Non-Safety Related ITS Applications, European Telecommunications Standard Institute (ETSI), Technical Report, Sophia Antipolis, France.

7. The Car-to-Car Communication Comsortium (C2CC).

8. Mecklenbräuker, C., Molisch, A., Karedal J., Tufvesson F., Paier A., Bernado L., Zemen T., Klemp O., \& Czink N. (2011). Vehicular channel characterization and its implications for wireless system design and performance. IEEE Proceedings, 99(7), pp. 1189-1212.

9. Ghafoor, K. Z., Bakar, K. A., Lloret, J., Khokhar, R. H., \& Lee, K. C. (2013). Intelligent beaconless geographical routing for urban vehicular environments. International Journal of Wireless Networks, 19(3), pp. 345-362.

10. Ghafoor, Lloret, J., K. Z., Bakar, Sadiq, A. S, \& Mussa, S. A. B. (2013). Beaconing approaches in vehicular ad hoc networks: a survey. International Journal of Wireless Personal Communications. Published Online (May 2013).

11. Michelson D. G., \& Ghassemzadeh S. S. (2009). New directions in wireless communications, Springer Science+Busines Media (Chapter 1).

12. IEEE 802.11p. (2010). Wireless LAN Medium Access Control (MAC) and Physical Layer (PHY) Specifications Amendment 6: Wireless Access in Vehicular Environments, Institute of Electrical and Electronic Engineers (IEEE), New York, USA.

13. Karedal, J., Czink, N., Paier, A., Tufvesson, F., \& Moisch, A. F. (2011). Path loss modeling for vehicle-tovehicle communications. IEEE Transactions on vehicular technology, 60(1), 323-327.

14. Cheng L., Henty B., Stancil D., Bai F., \& Mudalige P. (2007). Mobile vehicle-to-vehicle narrow-band channel measurement and characterization of the $5.9 \mathrm{GHz}$ dedicated short range communication (DSRC) frequency band. IEEE Journal on Selected Areas in Communications, vol. 25(8), pp. 1501-1516.

15. Cheng, L., Henty, B., Cooper R., Stancil D., \& Bai F. (2008). Multi-path propagation measurements for vehicular networks at $5.9 \mathrm{GHz}$. IEEE Wireless Communications and Networking Conference, pp. 1239-1244.

16. Tan I., Tang W., Laberteaux K., \& Bahai N. (2008). Measurement and analysis of wireless channel impairments in dsrc vehicular communications. IEEE International Conference on Communications, pp. 48824888.

17. Campuzano, A. J., Fernández, H., Balaguer, D., Vila-Jiménez A., Bernardo-Clemente, B., Rodrigo-Peñarrocha, V. M., Reig, J., Valero-Nogueira, A., \& Rubio, L. (2012). Vehicular-to-vehicular channel characterization and measurement results. WAVES, 4(1), pp. 14-24.

18. Kunisch, J., \& Pamp J. (2008). Wideband car-to-car radio channel measurements and model at 5.9 GHz. IEEE 68th Vehicular Technology Conference, pp. 1-5.

19 Gozalvez, J., \& Sepulcre, M. (2007). Opportunistic technique for efficient wireless vehicular communications. IEEE Vehicular Technology Magazine, 2(4), pp. 33-39.

20 Zang, Y., Stibor, L., Orfanos, G., Guo, S., \& Reumerman, H. (2005). An error model for inter-vehicle communications in highway scenarios at $5.9 \mathrm{GHz}$. Proc. Int. Workshop on performance evaluation of wireless ad hoc, sensor, and ubiquitous networks, pp. 49-56. 